\title{
„Der rechte Flügel außen, der linke aber innen...“ (Mauric. VI 1/2, lin. 7)
}

\author{
Petr V.Shuvalov \\ St. Petersburg State University, \\ 7-9, Universitetskaya nab., St. Petersburg, 199034, Russian Federation; p.shuvalov@spbu.ru
}

For citation: Petr V.Shuvalov. „Der rechte Flügel außen, der linke aber innen...“ (Mauric. VI 1/2, lin. 7). Philologia Classica 2018, 13(2), 308-311. https://doi.org/10.21638/11701/spbu20.2018.211

The training of the cavalrymen called Scythian in the $6^{\text {th }}$ book of Ps.-Maurice's Strategikon consists in the circular movement of the cavalrymen, "when ... the right wing is outside, and the left inside". The main purpose of this movement of cavalrymen is "to enclose an appropriate space" where the supposed enemy stands. But quite strange is the fact that the cavalrymen ride around the enemy in a clockwise direction: in this type of encirclement, the riders will turn to the enemy their right side not protected by the shield. There is a parallel locus in the 3rd book which is a condensed summary of the 'Scythian' chapter. There is another similar text in the 12th book, but this time with tactically correct movement of the cavalry, which is counter-clockwise. At the end of the description of this manoeuvre it is written that this manoeuvre is in use among the Scythians. To explain this following construction is proposed: the 'Scythian' chapter was first copied to XII D (Phase 1); at that time, perhaps, was the text of the

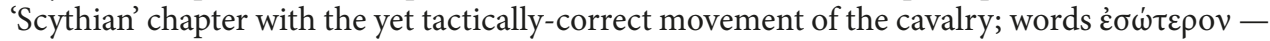
$\dot{\varepsilon} \xi \dot{\omega} \tau \varepsilon \rho 0 v$ were changed by their places as a result of the mental error of the copyist (Phase 2a);

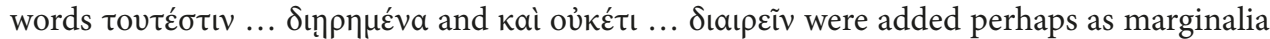
(Phase 2b); the newly revised text of the 'Scythian' chapter became source for III. 5 (Phase 3).

Keywords: Strategikon of Pseudo-Maurice, textology, military tactics, Scythians.

Im 6. Buch des Strategikons des sog. Pseudo-Maurikios ${ }^{1}$ handelt es sich um die Kavallerieübungen. Eine von denen, die „skythische Übung“ (VI $1 / 2^{2}$ ), wird als kreisförmiger Aufmarsch der Reiter beschrieben, wobei die Flügel im kreismäßigen Manöver einen „geeigneten Raum“ umfassen „der rechte Flügel nach außen, der linke nach innen vorgehend". Wozu genau dient das Manöver, wird im Weiteren nicht geklärt, aber allem Anschein nach besteht das Ziel eben darin, einen „geeigneten Raum“ zu umzingeln, in welchem sich der vermeintliche Feind befinden soll. Als sehr merkwürdig erscheint indes die Tatsache, dass die Kavalleristen des inneren Kreises diesen Raum im Uhrzeigersinn

${ }^{1}$ Der Text ist in mehreren Handschriften erhalten, die auf drei unziale (!) Klassen zurückgehen; dazu kommt die von allen drei Klassen abhängige Paraphrase. Die Endfassung (= Archetyp) wird in die Jahren 592-628 datiert (Dennis 1981, 15-16). In seinen Vorarbeiten stellt der Verf. eine Hypothese auf, Strategikon sei ein vielschichtiger Text gewesen, dessen Geschichte entsprechend als vielstufig aufgefasst werden muss (hierzu bspw. Shuvalov 2015b; 2015c). Ähnliche Ideen wurden bereits von einigen Forschern entwickelt: Vári 1906, 80-81; Darkó 1915, 14-15; 1937, 120-129; Kollautz 1985, 90, 92-93, cf. 108.

2 Der Text des Strategikons wird folgenderweise zitiert: Außer der Kapitel- sowie der Zeilennummerierung der 1981-Ausgabe von Dennis, wird auch die Paragraphen-Nummerierung der vorherigen Ausgabe (Mihăescu 1970) gegeben (z Bsp. I 1 lin.5 Dennis = I 1, 2 Mihăescu). Wenn sich die Kapitel-Nummer in den indizierten Ausgaben unterscheiden, werden sie mit Querstrich getrennt (13/12 [Dennis/Mihăescu]). 
umreiten, denn dabei werden sie ihre rechte, ungeschützte, nicht mit Schild gedeckte Seite dem Gegner bieten.

Nun gibt es im 3. Buch, am Ende der langen Beschreibung verschiedenartiger Aufstellungen und Übungen einer Schwadron einen Passus, welcher mit dem „skythischen“ Kapitel des 6. Buches gewisse Ähnlichkeit aufweist. Angeblich hatte der Autor dasselbe Manöver im Sinn. Unten wird der Textvergleich tabellarisch dargestellt.

Tabelle 1. Vergleich der relevanten Abschnitte des 6. und 3. Buches

\begin{tabular}{|c|c|}
\hline VI $1 / 2 \operatorname{lin} .2-8^{3}$ & III 5, 17 lin. $105-109^{4}$ \\
\hline 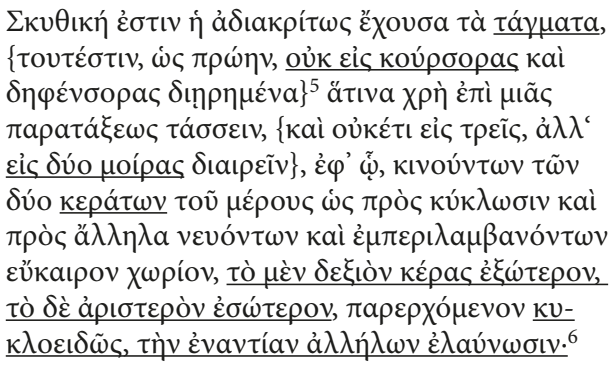 & 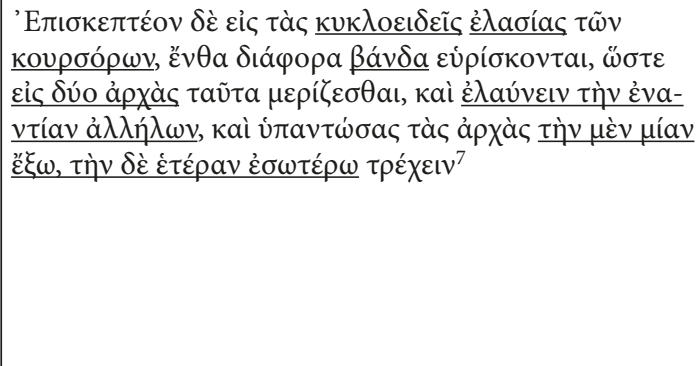 \\
\hline
\end{tabular}

Es ist kaum daran zu zweifeln, dass das zitierte Segment des 3. Buches eine Art Zusammenfassung des 'skythischen' Kapitels darstellt.

Darüber hinaus sei auf die Parallelbeschreibung im 12. Buch verwiesen, diesmal allerdings mit taktisch-richtiger Bewegung der Kavalleristen, will sagen entgegen der Uhrzeigerrichtung. Im letzten sich mit der Jagd befassenden Kapitel wird das UmkreisungManöver geschildert (XII D/10, 26-31. 2-8 lin. 55-111). Dort steht es klar und deutlich geschrieben: „Der rechte Flügel innen, der linke außen“ (XII D/10, 29 lin. 73-74). Am Ende der Schilderung liest man, dass eine solche Umkreisung „der Kampfaufstellung, welche die Skythen ausüben, ähnlich ist“. Der unten angegebene Textvergleich wird die Gemeinsamkeiten deutlicher machen.

${ }^{3}$ Die Handschriften enthalten nur eine Sonderlesart - $\dot{\varepsilon} \lambda a v ́ v \omega \sigma \iota v$ MLpLt vs $\dot{\varepsilon} \lambda a v ́ v o v \sigma ı v$ VNPA (nach Dennis 1981, 218).

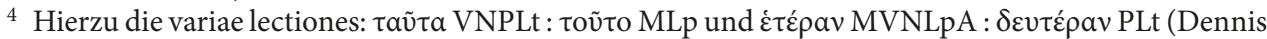
1981, 162).

${ }^{5}$ Die geschweiften Klammern \{\} grenzen die Wörter ab, die m. E. eine relativ spätere Ergänzung darstellen (dazu: Shuvalov 2015a).

6 „Die skythische (Aufstellung) hat die Schwadronen ohne Unterscheidung, d. h. wie früher, nicht differenziert in Angriffs- und Schutztruppen; man muss sie in einer Schlachtlinie aufstellen, sie dagegen in zwei Regimenter teilen, nicht aber in drei, damit heim kreisförmigen Aufmarsch der Division, wenn die beiden Flügel sich zur Einkreisung einander zuneigen und einen geeigneten Raum umfassen, der rechte Flügel außen, der linke aber innen, beide Flügel einander entgegen ziehen." (Alle Übersetzungen sind von E. Gammilscheg, s. Dennis 1981.)

7 „Bei den kreisförmigen Bewegungen der Angriffstruppen, an denen verschiedene Schwadronen teilnehmen, muss man beachten, dass sie auf zwei Kommandos aufgeteilt werden und gegeneinander reiten und bei der Begegnung das eine außen, das andere aber innen reitet, damit die Reiter nicht zusammenstoßen." 
Tabelle 2. Vergleich der relevanten Abschnitte des 6. und 12. Buches

\begin{tabular}{|c|c|}
\hline VI $1 / 2$ lin. $2-8$ & XII D/10, 28-30. 8 lin. $70-75.77 .104^{8}$ \\
\hline 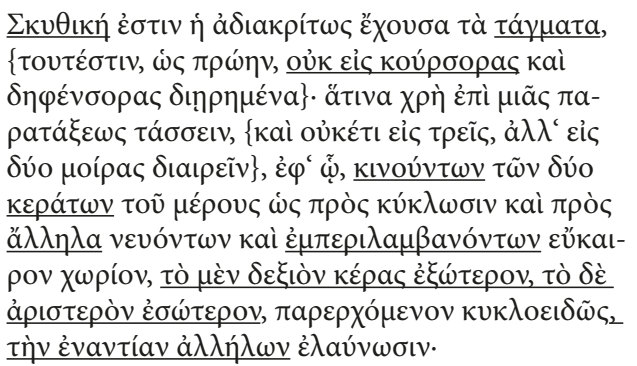 & 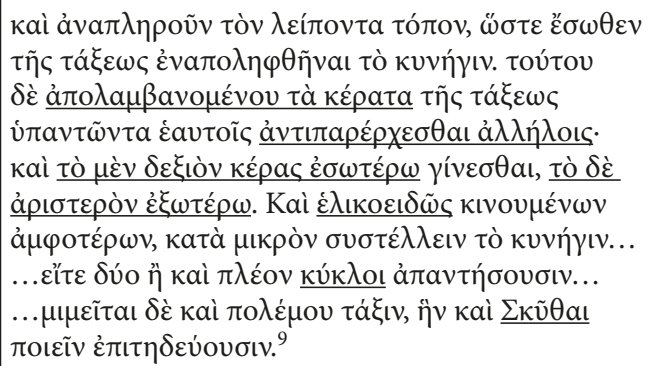 \\
\hline
\end{tabular}

Der Fehler $\grave{\varepsilon} \xi \hat{\omega} \tau \varepsilon \rho o v-\varepsilon \dot{\varepsilon} \sigma \omega ́ \tau \varepsilon \rho o v$ im Buch VI ist zwar leicht möglich und nicht gerade sehr selten. Angesichts der augenscheinlichen Gemeinsamkeiten lässt sich jedoch ein folgendes Entwicklungsschema vorstellen, welche (auch!) diesen Fehler besser erläutern könnte. Der Text des 'skythischen' Kapitels (VI 1/2 lin. 2-8) wurde zweimal als Grundlage für die relevanten Schilderungen gebraucht. Zunächst wurde er in das 'Jagdkapitel' des 12. Buches (XII D/10) aufgenommen, und zwar noch mit der taktisch richtigen Bewegung:

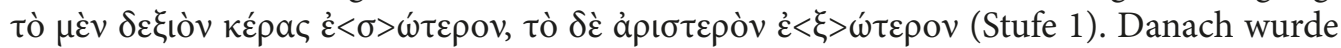
dieser ursprüngliche Text in den neuen Kodex übertragen, wobei die Begriffe $\dot{\varepsilon} \sigma \omega ́ \tau \varepsilon \rho o v$ und $\dot{\varepsilon} \xi \hat{\omega} \tau \varepsilon \rho o v$ als Folge eines Denkfehlers des Kopisten umgestellt wurden (Stufe 2a).

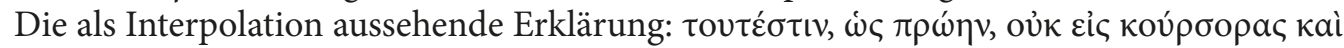

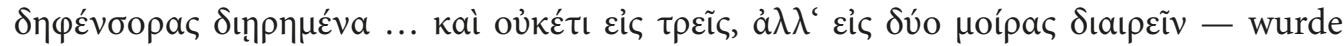
dann als Marginalnotiz ergänzt. Schließlich (Stufe 3) diente der neu revidierte Text des Skythen-Kapitels als Basis für die entsprechende Passage des 3. Buches (III 5, 17).

\section{Literaturhinweise}

Darkó E. Quelle foi doit-on ajouter à la Tactique de Léon le Sage. Budapest, 1915, 14-15.

Darkó E. Influences touraniennes sur l'évolution de l’art militaire des Grecs, des Romains et des Byzantins, 2. Byzantion 1937, 12, 119-137.

Dennis G. T. (Hg.), Gamillscheg E. (Übers.) Mauricii Strategicon. Wien, Verlag der Österreichischen Akademie der Wissenschaften, 1981.

Kollautz A. Das militärwissenschaftliche Werk des sogenannten Maurikios. Byzantiaka 1985, 5, 87-136.

Mihăescu H. (ed.). Mauricius. Arta militară. București, editura Academiei Republicii socialiste România, 1970.

Shuvalov P. German, kuzen Iustiniana, i „Strategikon“ Psevdo-Mavrikiia [Germanus, cousin of Justinian and the Strategikon of Pseudo-Maurice] (in Russian). Vizantiyskiy Vremennik 2015a, 74(99), 61-70.

Shuvalov P. Raznye psevdo-skify v "Strategikone Mavrikiia" [Diverse Scythians in the Pseudo-Maurices Strategikon] (in Russian), in: Skifiia: obraz i istoriko-kul'turnoe nasledie. Materialy konferentsii 2628 oktiabria 2015 goda: Institut vseobshchey istorii RAN. Moskva, 2015b, 111-116. $\mathrm{M}$ und $\mathrm{A}$.

${ }^{8}$ Hier ist der Originaltext nicht so gut erhalten: für das Kap. XII D/10 gibt es nur zwei Handschriften

9 „...und füllen den Zwischenraum aus, so dass das Wild innerhalb der Formation eingeschlossen verbleibt. Ist das aber durchgeführt, marschieren die Flügel der Schlachtordnung einander entgegen, der rechte Flügel innen, der linke außen. Sie bewegen sich spiralenförmig, ziehen kleinweise das Jagdgebiet zusammen ... ob zwei oder mehr Kreise einander begegnen, ... Man ahmt (damit) aber auch die Kampfaufstellung nach, die die Skythen anstreben." 
Shuvalov P. Raznye slaviane v "Strategikone" Psevdo-Mavrikiia [Diverse Slavs in the Pseudo-Maurice's Strategikon] (in Russian with English summary). Stratum-plus 2015c, 5, 217-223.

Vári R. Zur Überlieferung griechischer Taktiker. Byzantinische Zeitschrift 1906, 15, 47-87.

Received: July 31, 2018 Accepted: October 7, 2018

«Правое крыло снаружи, а левое внутри...» (Mauric. VI, 1/2 lin. 7)

\section{Петр Валерьевич Шувалов}

Санкт-Петербургский государственный университет,

Российская Федерация, 199034, Санкт-Петербург, Университетская наб., 7-9; p.shuvalov@spbu.ru

Упражнение кавалеристов, называемое «скифским» в VI книге «Стратегикона» ПсевдоМаврикия, состоит в круговом обходе, «когда... правое крыло снаружи, а левое внутри» совершает движение вокруг условной точки на местности, обозначенной как «подходящее пространство». Цель маневра - окружить пространство, где в реальности стоит предполагаемый враг. Довольно странным представляется то, что в тексте кавалеристы двигаются вокруг врага по часовой стрелке: при таком типе окружения всадники обращаются к врагу своей правой стороной, не защищенной щитом. Существует параллельный пассаж кн. III, представляющий собой сжатое резюме «скифской» главы, а также другой аналогичный текст в кн. XII, но с тактически правильным движением кавалерии, а именно против часовой стрелки. В конце описания указано, что этот маневр используется среди скифов. Предположительно «скифская» глава была скопирована в XII D, причем текст «скифской» главы описывал тактически верное движение

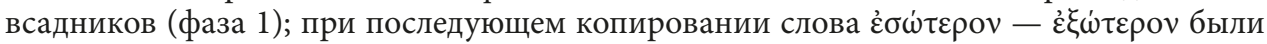
поменяны переписчиком местами в результате ошибки внутреннего диктанта (фаза 2);

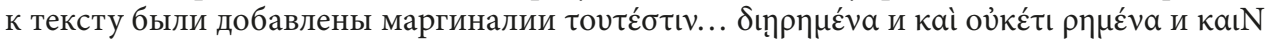

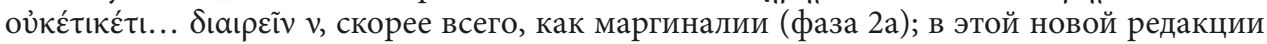
текст «скифской» главы стал источником для раздела III 5 (фаза 3).

Ключевые слова: Стратегикон Псевдо-Маврикия, текстология, военная тактика, скифы. 